\title{
On Characterizations of Fourier Frames and Tilings
}

\author{
Dao-Xin Ding ${ }^{1,2}$ and Hai-Xiong $\mathrm{Li}^{2}$ \\ ${ }^{1}$ Department of Mathematics, Hubei University, Wuhan 430062, China \\ ${ }^{2}$ School of Mathematics and Quantitative Economics, Hubei University of Education, Wuhan 430205, China
}

Correspondence should be addressed to Hai-Xiong Li; haixiongli@sina.com

Received 20 March 2013; Accepted 29 April 2013

Academic Editor: Morteza Rafei

Copyright (c) 2013 D.-X. Ding and H.-X. Li. This is an open access article distributed under the Creative Commons Attribution License, which permits unrestricted use, distribution, and reproduction in any medium, provided the original work is properly cited.

We give some characterizations of Fourier frames and tilings and obtain a more general form of characterizations of spectra and tilings.

\section{Introduction}

A countable family of elements $\left\{f_{n}\right\}_{n \in \mathbb{N}}$ in a separable Hilbert space $H$ is called a frame if there are positive constants $A, B$ such that

$$
A\|f\|^{2} \leq \sum_{n \in \mathbb{N}}\left|\left\langle f, f_{n}\right\rangle\right|^{2} \leq B\|f\|^{2}
$$

for all $f \in H$. $A$ and $B$ are called frame bounds. The sequence is called a tight frame if $A=B$. The sequence is called Bessel if the second inequality above holds. In this case, $B$ is called the Bessel bound. Frames were first introduced by Duffin and Schaeffer [1] in the context of nonharmonic Fourier series, and today they have applications in a wide range of areas. A frame can be considered as a generalized basis in the sense that every element in $H$ can be written as a linear combination of the frame elements.

In this paper, we consider Fourier frames for a special separable Hilbert space. Let $\Omega \subset \mathbb{R}^{d}$ have positive Lebesgue measure $m(\Omega)>0$ and let $\Lambda$ be a discrete subset of $\mathbb{R}^{d}$. The inner product and the norm on $L^{2}(\Omega)$ are

$$
\begin{gathered}
\langle f(x), g(x)\rangle_{\Omega}=\frac{1}{m(\Omega)} \int_{\Omega} f(x) \overline{g(x)} d x, \\
\|f\|_{\Omega}^{2}=\frac{1}{m(\Omega)} \int_{\Omega}|f(x)|^{2} d x .
\end{gathered}
$$

We write

$$
\begin{gathered}
e_{\lambda}(x):=e^{2 \pi i\langle\lambda, x\rangle} \quad \text { for } x \in \mathbb{R}^{d}, \\
\mathscr{E}(\Lambda):=\left\{e_{\lambda}(x): \lambda \in \Lambda\right\} .
\end{gathered}
$$

If $\mathscr{E}(\Lambda)$ is a frame or an orthonormal basis for $L^{2}(\Omega)$, then $\mathscr{E}(\Lambda)$ and $(\Omega, \Lambda)$ are called a Fourier frame and a spectral pair, respectively. In the case of the spectral pair, the $\Lambda$ is then called a spectrum for $\Omega$ and $\Omega$ is called a spectral set. We follow the terminology of [2] and consider the packing and tiling in $\mathbb{R}^{d}$ by compact set $\Omega$ of the following kind.

A compact set $\Omega$ in $\mathbb{R}^{d}$ is a regular region if it has positive Lebesgue measure, is the closure of its interior $\Omega^{\circ}$, and has a boundary $\partial \Omega=\Omega \backslash \Omega^{\circ}$ of measure zero. If $\Omega$ is a regular region, then a discrete set $\Lambda$ is a packing set for $\Omega$ if the sets $\{\Omega+\lambda: \lambda \in \Lambda\}$ have disjoint interiors or the intersections $(\Omega+\lambda) \cap(\Omega+\mu)$ for $\lambda \neq \mu$ in $\Lambda$ have measure zero. It is a tiling set if, further, the translates $\{\Omega+\lambda: \lambda \in \Lambda$ \} cover $\mathbb{R}^{d}$ up to measure zero. In these cases, we say that $\Omega+\Lambda$ is a packing or tiling of $\mathbb{R}^{d}$, respectively. Equivalently, we call $(\Omega, \Lambda)$ a packing pair or a tiling pair, respectively.

It is well known that spectral sets and tilings are connected by the following conjecture of Fuglede [3].

Spectral Set Conjecture. A set $\Omega$ in $\mathbb{R}^{d}$ is a spectral set if and only if it tiles $\mathbb{R}^{d}$ by translations.

Many people attempt to prove the spectral set conjecture for some special sets, although the conjecture is false in many 
cases (see [4-7]). For example, Jorgensen and Pedersen [8] conjectured that $\left([0,1]^{n}, \Lambda\right)$ is a spectral pair if and only if $\left([0,1]^{n}, \Lambda\right)$ is a tiling pair. They established the conjecture for dimension $n \leq 3$ and for all $n$ when $\Lambda$ is a discrete periodic set. Iosevich and Pedersen [9] simultaneously and independently established the above-mentioned conjecture by a different approach based on a geometric argument. Kolountzakis [10] gave an alternative proof of this fact, which is based on a characterization of translational tiling by a Fourier analytic criterion. Lagarias et al. [2] related the spectra of sets $\Omega$ to tiling in the Fourier space and obtained the following characterization of spectra and tilings.

Theorem 1. Let $\Omega$ be a regular region in $\mathbb{R}^{d}$ and let $\Lambda$ be such that the set of exponentials $\mathscr{E}(\Lambda)$ is orthogonal for $L^{2}(\Omega)$. Suppose that $D$ is a regular region with $m(\Omega) m(D)=1$ such that $D+\Lambda$ is a packing of $\mathbb{R}^{d}$. Then, $\Lambda$ is a spectrum for $\Omega$ if and only if $D+\Lambda$ is a tiling of $\mathbb{R}^{d}$.

Li [11] presented an elementary approach to obtain a more general form of Theorem 1. Enlightened by the ideas from $[11,12]$, we give some characterizations of Fourier frames and tilings and extend several results in [2] and [11].

\section{Main Results and Their Proofs}

Throughout this section, let $\Omega$ and $D$ be two regular regions in $\mathbb{R}^{d}$. By the definition of frames, we may get that the following lemma.

Lemma 2. Let $\Delta \subset \mathbb{R}^{d}$ be a discrete set. If $\mathscr{E}(\Delta)$ is a frame for $L^{2}(\Omega)$ with frame bounds $A, B$, then

$$
A(m(\Omega))^{2} \leq \sum_{\delta \in \Delta}\left|\widehat{\chi_{\Omega}}(t-\delta)\right|^{2} \leq B(m(\Omega))^{2}, \quad \forall t \in \mathbb{R}^{d},
$$

where

$$
\widehat{\chi_{\Omega}}(u)=\int_{\mathbb{R}^{d}} \chi_{\Omega}(x) e^{-2 \pi i\langle u, x\rangle} d x, \quad u \in \mathbb{R}^{d}
$$

is the Fourier transform of the characteristic function $\chi_{\Omega}(x)$.

Proof. By the frame inequality (1), for any $t \in \mathbb{R}^{d}$, we have

$$
\begin{aligned}
\sum_{\delta \in \Delta} \mid \widehat{\chi_{\Omega}} & \left.(t-\delta)\right|^{2} \\
& =\sum_{\delta \in \Delta}\left|m(\Omega)\left\langle e_{t}(x), e_{\delta}(x)\right\rangle_{\Omega}\right|^{2} \\
& \leq B(m(\Omega))^{2}\left\|e_{t}(x)\right\|_{\Omega}^{2}=B(m(\Omega))^{2} .
\end{aligned}
$$

Similarly, we get $A(m(\Omega))^{2} \leq \sum_{\delta \in \Delta}\left|\widehat{\chi_{\Omega}}(t-\delta)\right|^{2}$.

Remark 3. In the case for $A=B$, if $\mathscr{E}(\Delta)$ is a tight frame for $L^{2}(\Omega)$ with the frame bound $B$, then

$$
\sum_{\delta \in \Delta}\left|\widehat{\chi_{\Omega}}(t-\delta)\right|^{2}=B(m(\Omega))^{2}, \quad \forall t \in \mathbb{R}^{d} .
$$

If $\mathscr{E}(\Delta)$ is a Bessel sequence for $L^{2}(\Omega)$ with the Bessel bound $B$, then

$$
\sum_{\delta \in \Delta}\left|\widehat{\chi_{\Omega}}(t-\delta)\right|^{2} \leq B(m(\Omega))^{2}, \quad \forall t \in \mathbb{R}^{d}
$$

Moreover, $\mathscr{E}(\Delta)$ is an orthonormal basis for $L^{2}(\Omega)$ if and only if

$$
\sum_{\delta \in \Delta}\left|\widehat{\chi_{\Omega}}(t-\delta)\right|^{2}=(m(\Omega))^{2}, \quad \forall t \in \mathbb{R}^{d}
$$

Since $\left|\widehat{\chi_{\Omega}}(u)\right|=\left|\widehat{\chi_{\Omega}}(-u)\right|$, for all $u \in \mathbb{R}^{d}$, if we substitute "-" for “+” in (4), (7), (8), and (9), all the above results also hold.

In the remainder of this paper, we assume that $\Theta \subset \mathbb{R}^{d}$ is a discrete subset and $\Lambda$ and $\Gamma$ are two finite subsets of $\mathbb{R}^{d}$ such that $\Theta+\Lambda$ and $\Theta+\Gamma$ are two direct sums.

Theorem 4. If $\mathscr{E}(\Theta+\Lambda)$ is a frame for $L^{2}(\Omega)$ with frame bounds $A, B$, and $(D, \Theta+\Gamma)$ is a tiling pair, then

$$
\frac{\# \Lambda}{B \# \Gamma} \leq m(\Omega) m(D) \leq \frac{\# \Lambda}{A \# \Gamma},
$$

where \# denotes the cardinality of some set.

Proof. Let $\Lambda:=\left\{\lambda_{1}, \lambda_{2}, \ldots, \lambda_{p}\right\}$ and $\Gamma:=\left\{\gamma_{1}, \gamma_{2}, \ldots, \gamma_{q}\right\}$ with $\# \Lambda=p$ and $\# \Gamma=q$. Since $\mathscr{E}(\Theta+\Lambda)$ is a frame for $L^{2}(\Omega)$ with frame bounds $A, B$, it follows from Lemma 2 that

$$
\begin{array}{r}
A(m(\Omega))^{2} \leq \sum_{i=1}^{p} \sum_{\theta \in \Theta}\left|\widehat{\chi_{\Omega}}\left(t+\theta+\lambda_{i}\right)\right|^{2} \leq B(m(\Omega))^{2}, \\
\forall t \in \mathbb{R}^{d} .
\end{array}
$$

Note that $(D, \Theta+\Gamma)$ is a tiling pair, from the Plancherel's formula on $L^{2}\left(\mathbb{R}^{d}\right)$, we have the following:

$$
\begin{aligned}
m(\Omega) & =\left\|\chi_{\Omega}\right\|_{\mathbb{R}^{d}}^{2}=\left\|\widehat{\chi_{\Omega}}\right\|_{\mathbb{R}^{d}}^{2}=\int_{\mathbb{R}^{d}}\left|\widehat{\chi_{\Omega}}(t)\right|^{2} d t \\
& =\frac{1}{p} \int_{\mathbb{R}^{d}} \sum_{i=1}^{p}\left|\widehat{\chi_{\Omega}}\left(t+\lambda_{i}\right)\right|^{2} d t \\
& =\frac{1}{p} \int_{\cup_{\theta \in \Theta, 1 \leq j \leq q}\left(D+\theta+\gamma_{j}\right)} \sum_{i=1}^{p}\left|\widehat{\chi_{\Omega}}\left(t+\lambda_{i}\right)\right|^{2} d t \\
& =\frac{1}{p} \sum_{j=1}^{q} \sum_{\theta \in \Theta} \int_{D} \sum_{i=1}^{p}\left|\widehat{\chi_{\Omega}}\left(t+\theta+\lambda_{i}+\gamma_{j}\right)\right|^{2} d t \\
& =\frac{1}{p} \sum_{j=1}^{q} \int_{D} \sum_{\theta \in \Theta} \sum_{i=1}^{p}\left|\widehat{\chi_{\Omega}}\left(t+\theta+\lambda_{i}+\gamma_{j}\right)\right|^{2} d t \\
& =\frac{1}{p} \sum_{j=1}^{q} \int_{D} \sum_{i=1}^{p} \sum_{\theta \in \Theta}\left|\widehat{\chi_{\Omega}}\left(t+\theta+\lambda_{i}+\gamma_{j}\right)\right|^{2} d t \\
& \leq \frac{q}{p} B(m(\Omega))^{2} m(D) .
\end{aligned}
$$


The bottom third equality holds by Lebesgue dominated convergence theorem and the last inequality follows from (11). Thus, $p / q B \leq m(\Omega) m(D)$. Similarly, we get $m(\Omega) m(D) \leq$ $p / q A$. Hence, the proof is completed.

Since an orthonormal basis is also a tight frame with frame bounds $A=B=1$, we get the following corollary.

Corollary 5. If $\mathscr{E}(\Theta+\Lambda)$ is an orthonormal basis for $L^{2}(\Omega)$, and $(D, \Theta+\Gamma)$ is a tiling pair, then $m(\Omega) m(D)=\# \Lambda / \# \Gamma$.

Lemma 6. Let $\Theta+\Lambda$ be such that the set of exponentials $\mathscr{E}(\Theta+$ $\Lambda)$ is a Bessel sequence for $L^{2}(\Omega)$ with the Bessel bound B. If $D+\Theta+\Gamma$ is a tiling of $\mathbb{R}^{d}$ with $m(\Omega) m(D) \leq \# \Lambda / B \# \Gamma$, then

$$
\sum_{\lambda \in \Lambda} \sum_{\theta \in \Theta}\left|\widehat{\chi_{\Omega}}(t+\theta+\lambda)\right|^{2}=B(m(\Omega))^{2}, \quad \forall t \in \mathbb{R}^{d} .
$$

Proof. Keep the assumptions on $\Lambda$ and $\Gamma$ in the above proof. Since $\mathscr{E}(\Theta+\Lambda)$ is a Bessel sequence for $L^{2}(\Omega)$ with the Bessel bound $B$, it follows from Remark 3 that

$$
\sum_{i=1}^{p} \sum_{\theta \in \Theta}\left|\widehat{\chi_{\Omega}}\left(t+\theta+\lambda_{i}\right)\right|^{2} \leq B(m(\Omega))^{2}, \quad \forall t \in \mathbb{R}^{d}
$$

Since $D+\Theta+\Gamma$ is a tiling of $\mathbb{R}^{d}$ and $m(\Omega) m(D) \leq \# \Lambda / B \# \Gamma=$ $p / q B$, for any $y \in \mathbb{R}^{d}$, then we have

$$
\begin{aligned}
m(\Omega) & =\int_{\mathbb{R}^{d}}\left|\widehat{\chi_{\Omega}}(t)\right|^{2} d t \\
& =\frac{1}{p} \int_{\mathbb{R}^{d}} \sum_{i=1}^{p}\left|\widehat{\chi_{\Omega}}\left(t+\lambda_{i}\right)\right|^{2} d t \\
& =\frac{1}{p} \int_{\cup_{\theta \in \Theta, 1 \leq j \leq q}\left(D+y+\theta+\gamma_{j}\right)} \sum_{i=1}^{p}\left|\widehat{\chi_{\Omega}}\left(t+\lambda_{i}\right)\right|^{2} d t \\
& =\frac{1}{p} \sum_{j=1}^{q} \sum_{\theta \in \Theta} \int_{D+y} \sum_{i=1}^{p}\left|\widehat{\chi_{\Omega}}\left(t+\theta+\lambda_{i}+\gamma_{j}\right)\right|^{2} d t \\
& =\frac{1}{p} \sum_{j=1}^{q} \int_{D+y} \sum_{\theta \in \Theta} \sum_{i=1}^{p}\left|\widehat{\chi_{\Omega}}\left(t+\theta+\lambda_{i}+\gamma_{j}\right)\right|^{2} d t \\
& =\frac{1}{p} \sum_{j=1}^{q} \int_{D+y} \sum_{i=1}^{p} \sum_{\theta \in \Theta}\left|\widehat{\chi_{\Omega}}\left(t+\theta+\lambda_{i}+\gamma_{j}\right)\right|^{2} d t \\
& \leq \frac{q}{p} B(m(\Omega))^{2} m(D) \leq m(\Omega),
\end{aligned}
$$

which yields

$$
\sum_{i=1}^{p} \sum_{\theta \in \Theta}\left|\widehat{\chi_{\Omega}}\left(t+\theta+\lambda_{i}\right)\right|^{2}=B(m(\Omega))^{2}
$$

for almost every $t$ in $D+y$. Since $y$ is arbitrary, (16) holds for almost every $t$ in $\mathbb{R}^{d}$. By the continuity of the function on the left side of (16), we see that (16) holds for every $t$ in $\mathbb{R}^{d}$.
Theorem 7. If $\mathscr{E}(\Theta+\Lambda)$ is orthogonal in $L^{2}(\Omega)$ and $(D, \Theta+\Gamma)$ is a tiling pair with $m(\Omega) m(D)=\# \Lambda / \# \Gamma$, then $\mathscr{E}(\Theta+\Lambda)$ is an orthonormal basis for $L^{2}(\Omega)$.

Proof. The proof is straightforward by the above lemma.

Theorem 8. Suppose that $D+\Theta+\Gamma$ is a packing of $\mathbb{R}^{d}$ with $\# \Lambda / A \# \Gamma \leq m(\Omega) m(D)$. If $\mathscr{E}(\Theta+\Lambda)$ is a frame for $L^{2}(\Omega)$ with the frame bounds $A, B$, then $D+\Theta+\Gamma$ is a tiling of $\mathbb{R}^{d}$.

Proof. Since $\mathscr{E}(\Theta+\Lambda)$ is a frame for $L^{2}(\Omega)$ with the frame bounds $A, B$, then (11) holds. If $D+\Theta+\Gamma$ is a packing of $\mathbb{R}^{d}$, then it follows from (11) and $\# \Lambda / A \# \Gamma \leq m(\Omega) m(D)$ that

$$
\begin{aligned}
m(\Omega) & =\int_{\mathbb{R}^{d}}\left|\widehat{\chi_{\Omega}}(t)\right|^{2} d t \\
& =\frac{1}{p} \int_{\mathbb{R}^{d}} \sum_{i=1}^{p}\left|\widehat{\chi_{\Omega}}\left(t+\lambda_{i}\right)\right|^{2} d t \\
& \geq \frac{1}{p} \int_{\cup_{\theta \in \Theta, 1 \leq j \leq q}\left(D+\theta+\gamma_{j}\right)} \sum_{i=1}^{p}\left|\widehat{\chi_{\Omega}}\left(t+\lambda_{i}\right)\right|^{2} d t \\
& =\frac{1}{p} \sum_{j=1}^{q} \sum_{\theta \in \Theta} \int_{D} \sum_{i=1}^{p}\left|\widehat{\chi_{\Omega}}\left(t+\theta+\lambda_{i}+\gamma_{j}\right)\right|^{2} d t \\
& =\frac{1}{p} \sum_{j=1}^{q} \int_{D} \sum_{\theta \in \Theta} \sum_{i=1}^{p}\left|\widehat{\chi_{\Omega}}\left(t+\theta+\lambda_{i}+\gamma_{j}\right)\right|^{2} d t \\
& =\frac{1}{p} \sum_{j=1}^{q} \int_{D} \sum_{i=1}^{p} \sum_{\theta \in \Theta}\left|\widehat{\chi_{\Omega}}\left(t+\theta+\lambda_{i}+\gamma_{j}\right)\right|^{2} d t \\
& \geq \frac{q}{p} A(m(\Omega))^{2} m(D) \geq m(\Omega) .
\end{aligned}
$$

Thus, $D+\Theta+\Gamma$ is a tiling of $\mathbb{R}^{d}$.

It is clear that the above theorem yields the following corollary.

Corollary 9. Suppose that $D+\Theta+\Gamma$ is a packing of $\mathbb{R}^{d}$ with $\# \Lambda / \# \Gamma=m(\Omega) m(D)$. If $\mathscr{E}(\Theta+\Lambda)$ is an orthonormal basis for $L^{2}(\Omega)$, then $D+\Theta+\Gamma$ is a tiling of $\mathbb{R}^{d}$.

Combining Theorem 7 with Corollary 9, we obtain a more general form of the theorem in [11] and Theorem 1.

Theorem 10. Suppose that $\mathscr{E}(\Theta+\Lambda)$ is orthogonal in $L^{2}(\Omega)$, and $(D, \Theta+\Gamma)$ is a packing pair with $m(\Omega) m(D)=\# \Lambda / \# \Gamma$. Then, $(\Omega, \Theta+\Lambda)$ is a spectral pair if and only if $(D, \Theta+\Gamma)$ is a tiling pair.

Example 11. Let $p, q$ be two positive integers. Take the following:

$$
\Omega=[0,1], \quad D=\left[0, \frac{p}{q}\right], \quad \Theta=\{p k: k \in \mathbb{Z}\},
$$




$$
\Lambda=\{0,1, \ldots, p-1\}, \quad \Gamma=\left\{0, \frac{p}{q}, \frac{2 p}{q}, \ldots, \frac{(q-1) p}{q}\right\} .
$$

We see that $m(\Omega) m(D)=\# \Lambda / \# \Gamma,(\Omega, \Theta+\Lambda)$ is a spectral pair and $(D, \Theta+\Gamma)$ is a tiling pair.

\section{Acknowledgment}

This work was supported by the National Natural Science Foundation of China 11271148 and 11271114.

\section{References}

[1] R. J. Duffin and A. C. Schaeffer, "A class of nonharmonic Fourier series," Transactions of the American Mathematical Society, vol. 72, pp. 341-366, 1952.

[2] J. C. Lagarias, J. A. Reeds, and Y. Wang, "Orthonormal bases of exponentials for the $n$-cube," Duke Mathematical Journal, vol. 103 , no. 1, pp. 25-37, 2000.

[3] B. Fuglede, "Commuting self-adjoint partial differential operators and a group theoretic problem," Journal of Functional Analysis, vol. 16, pp. 101-121, 1974.

[4] M. N. Kolountzakis and M. Matolcsi, "Complex Hadamard matrices and the spectral set conjecture," Collectanea Mathematica, pp. 281-291, 2006.

[5] M. N. Kolountzakis and M. Matolcsi, "Tiles with no spectra," Forum Mathematicum, vol. 18, no. 3, pp. 519-528, 2006.

[6] M. Matolcsi, "Fuglede's conjecture fails in dimension 4," Proceedings of the American Mathematical Society, vol. 133, no. 10, pp. 3021-3026, 2005.

[7] T. Tao, "Fuglede's conjecture is false in 5 and higher dimensions," Mathematical Research Letters, vol. 11, no. 2-3, pp. 251-258, 2004.

[8] P. E. T. Jorgensen and S. Pedersen, "Spectral pairs in Cartesian coordinates," The Journal of Fourier Analysis and Applications, vol. 5, no. 4, pp. 285-302, 1999.

[9] A. Iosevich and S. Pedersen, "Spectral and tiling properties of the unit cube," International Mathematics Research Notices, no. 16, pp. 819-828, 1998.

[10] M. N. Kolountzakis, "Packing, tiling, orthogonality and completeness," The Bulletin of the London Mathematical Society, vol. 32, no. 5, pp. 589-599, 2000.

[11] J.-L. Li, "Note on a characterization of spectra and tilings," Journal of Mathematical Analysis and Applications, vol. 289, no. 1, pp. 244-247, 2004.

[12] J.-L. Li, “On characterizations of spectra and tilings," Journal of Functional Analysis, vol. 213, no. 1, pp. 31-44, 2004. 


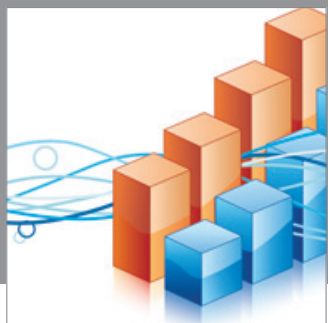

Advances in

Operations Research

mansans

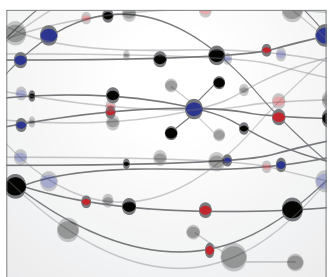

The Scientific World Journal
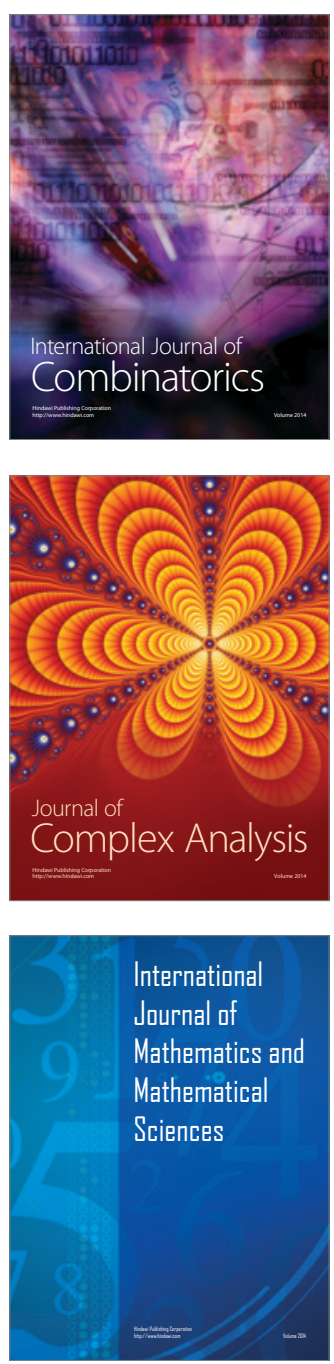
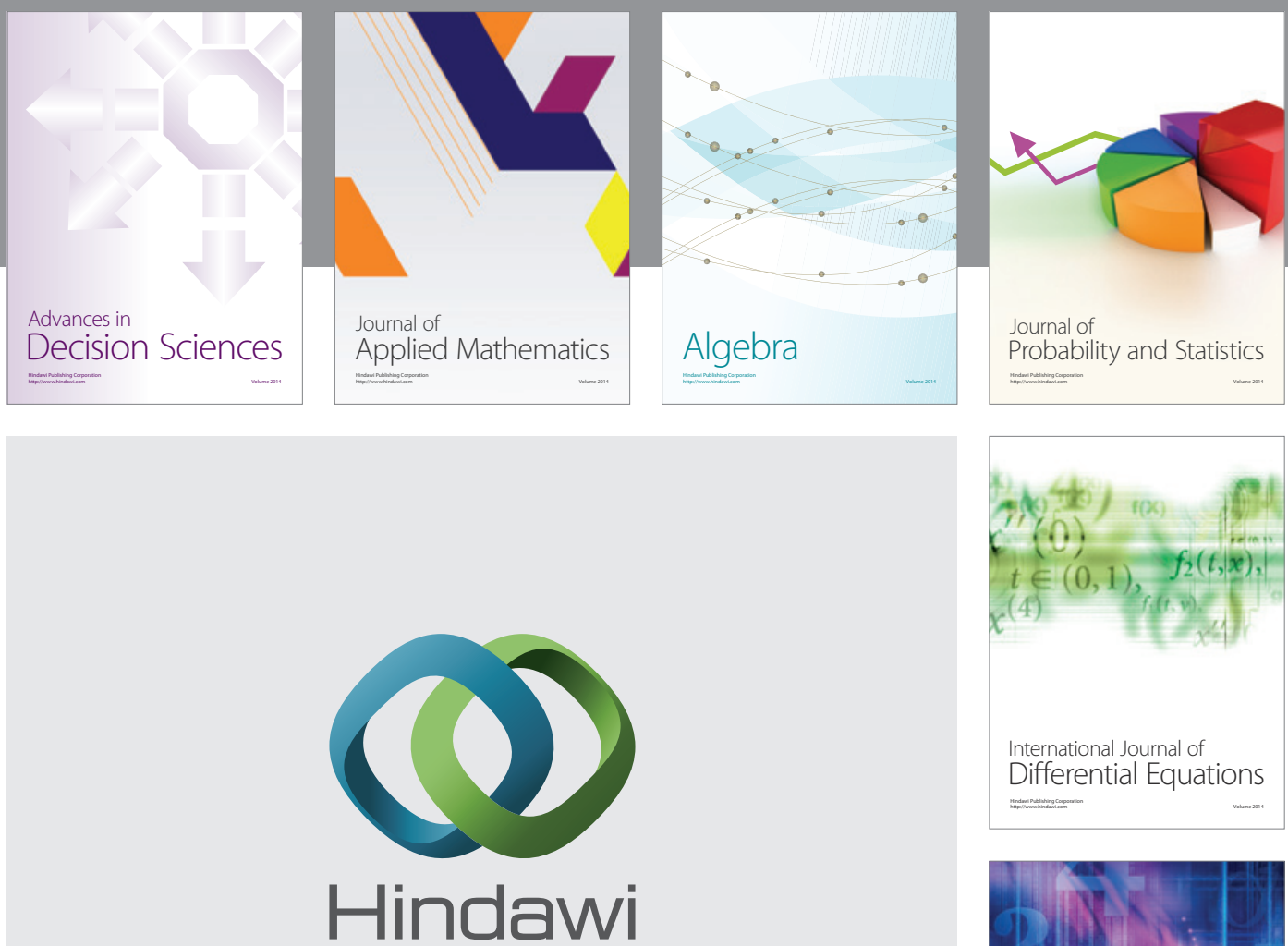

Submit your manuscripts at http://www.hindawi.com
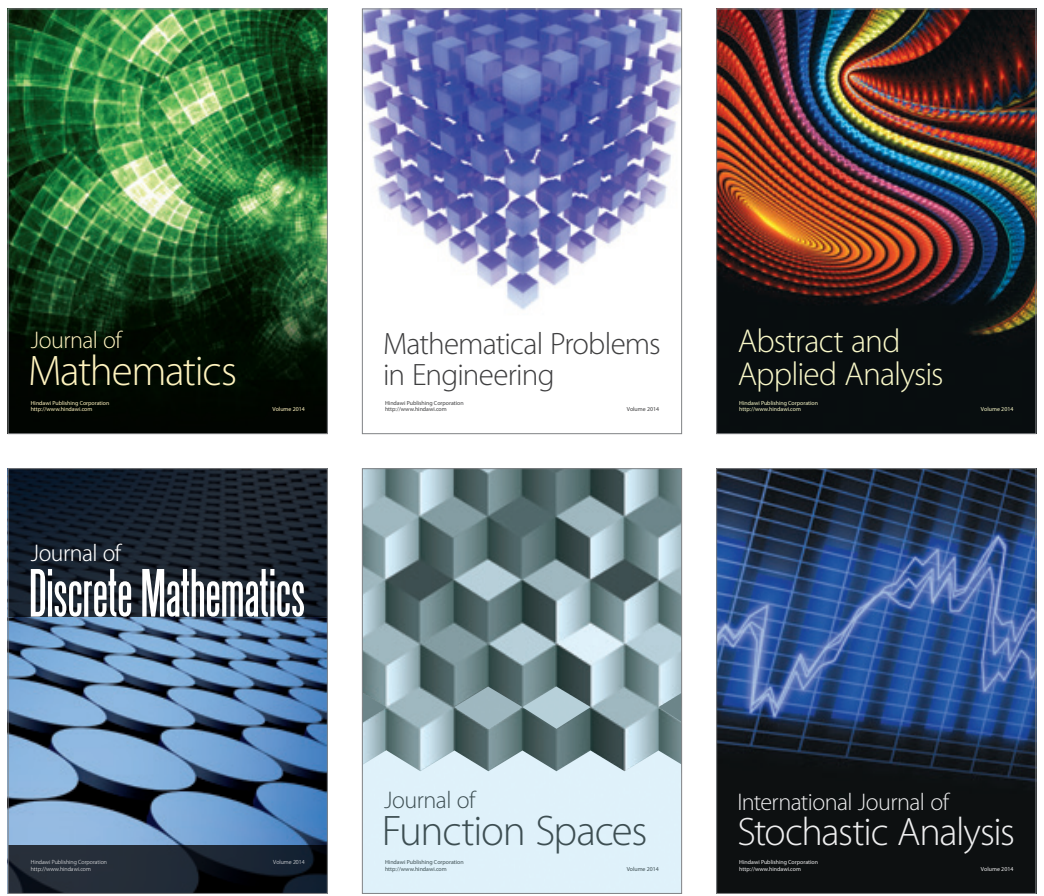

Journal of

Function Spaces

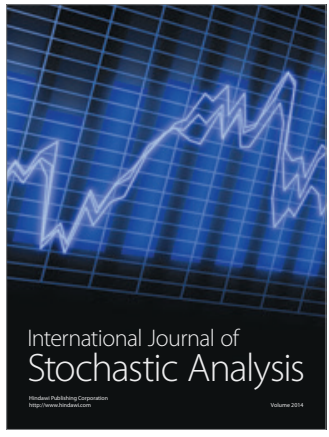

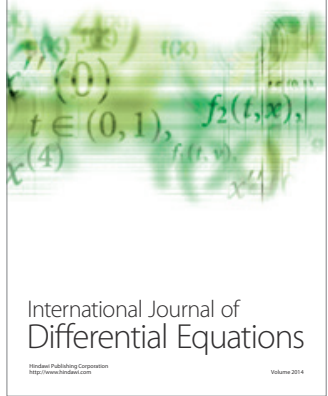
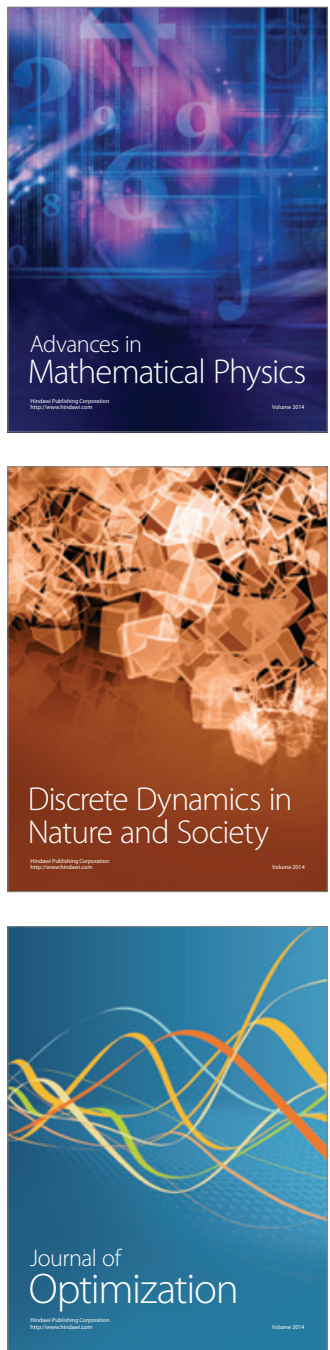